\title{
Pulse-forming dynamics of a cw passively mode-locked Ti:sapphire/DDI laser
}

\author{
Jahn-Chung Kuo, Jia-Min Shieh, Chin-Der Hwang, C.-S. Chang, and Ci-Ling Pan \\ Institute of Electro-optical Engineering, National Chiao Tung University, Hsinchu, Taiwan 300, China
}

Kaung-Hsiung Wu

Department of Electrophysics, National Chiao Tung University, Hsinchu, Taiwan 300, China

Received October 21, 1991

\begin{abstract}
The dynamics of pulse formation in a cw passively mode-locked Ti:sapphire/DDI laser has been investigated. It is found that the time required for the circulating energy in the cavity to build up and bleach the dye absorber is approximately $140 \mu \mathrm{s}$. A further $230 \mu \mathrm{s}$ is required for the buildup of steady-state picosecond pulses. Concurrently the laser output spectrum initially narrows in the cw regime, begins to broaden as the laser becomes modulated, and finally establishes the steady-state spectral distribution.
\end{abstract}

The solid-state nature, high saturation power, and broad bandwidth of Ti:sapphire have allowed it to become one of the most important laser crystals for the generation and amplification of ultrashort optical pulses. Much effort has been devoted to mode lock the Ti:sapphire laser using various schemes. These include additive-pulse mode locking, ${ }^{1}$ active mode locking, ${ }^{2,3}$ coupled-cavity resonant passive mode locking, ${ }^{4}$ and self-mode locking ${ }^{5,6}$ or Kerrlens mode locking. ${ }^{7}$ Passive mode locking has also been demonstrated by using organic dyes or semiconductor-doped glass as the saturable absorber. Pulses as short as 4 and $2.7 \mathrm{ps}$, respectively, have been obtained with a DDI dye absorber ${ }^{8}$ and a semiconductor-doped glass absorber. ${ }^{9}$ Using another absorber dye, HITCI, Sarukura et al..$^{10}$ achieved 15.4-ps chirped pulses and 2.4-ps compressed pulses with a grating-prism pair outside the cavity. A dispersion-compensating prism pair incorporated inside the same cavity has allowed them to produce $150-\mathrm{fs}$ pulses directly. Subsequent pulse compression yielded 50-fs pulses. ${ }^{11}$ Furthermore a colliding-pulse mode-locked Ti:sapphire/HITCI laser that uses an antiresonant ring configuration has generated 50 -fs pulses. ${ }^{12}$ These studies have concentrated on the steady-state characteristics, and relatively less attention has been paid to the pulseforming dynamics of mode-locked Ti:sapphire lasers. Goodberlet et al. ${ }^{13}$ showed that their selfstarting additive-pulse mode-locking Ti:sapphire laser evolved from mode-beating fluctuations to mode-locked operation in $\approx 200 \mu \mathrm{s}$, or 17,000 round trips. For a cw passively mode-locked Ti:sapphire/ HITCI laser system, Ishida et al. ${ }^{14}$ reported that the pulse train rapidly built up with a time constant of $\approx 200 \mu \mathrm{s}$ and reached the steady state on a time scale of milliseconds to seconds. Chen and Wang ${ }^{15}$ recently proposed a new model to describe the evolution of Ishida's laser. They showed that the time required to shorten the pulses from $5 \mathrm{~ns}$ to $100 \mathrm{fs}$
FWHM was $\sim 200-300 \mu \mathrm{s}$. The excited-state lifetimes of DDI and HITCI are quite different: $17 \mathrm{ps}$ and $1.2 \mathrm{~ns}$, respectively. ${ }^{16}$ It is interesting to compare the buildup time for steady-state short pulses of these two passively mode-locked lasers. In this Letter we report for the first time, to our knowledge, results on the temporal evolution of a $\mathrm{cw}$ passively mode-locked Ti:sapphire/DDI laser from cw operation to steady-state picosecond pulses. The evolution of the corresponding spectra of the laser output is also presented.

A block diagram of the experimental setup is shown in Fig. 1. The configuration of our passively mode-locked Ti:sapphire/DDI laser is shown in the inset of Fig. 1. The laser employs a $20-\mathrm{mm}$-long Brewster-angle-cut $\mathrm{Ti}: \mathrm{Al}_{2} \mathrm{O}_{3}$ crystal ( $0.05 \%$ doping) as the gain medium and an organic dye (DDI) as the saturable absorber. The cavity consists of six mirrors, with a round-trip time of $10 \mathrm{~ns}$, and a $1 \%$ transmission output coupler. No bandwidth-limiting or tuning elements were employed inside the cavity. The gain and absorber folding sections of the resonator had focusing mirrors of 15 - and 5 -cm radii of curvature, respectively. The saturable absorber DDI was dissolved in ethylene glycol with a concentration of $4 \times 10^{-4} \mathrm{M}$. A standard nozzle obtained from Coherent was used for the absorber jet. Pumped by an all-line 4.8-W cw argon-ion laser, our mode-locked Ti:sapphire laser has routinely generated optical pulses with average power of $70 \mathrm{~mW}$ and pulse durations of 3-5 ps (assuming a sech ${ }^{2}$ pulse shape). For investigating the dynamics of pulse formation in this laser, we used both a fast photodetector with an oscilloscope and the timegating technique previously described. ${ }^{17}$ The $\mathrm{cw}$ argon-ion laser was mechanically chopped to obtain $600-\mathrm{Hz}$ square pulses with a FWHM of $800 \mu$ s and a rise time of $30 \mu \mathrm{s}$. The Ti:sapphire laser output was fed to a noncollinear autocorrelator or a monochromator and detected by a gatable photomultiplier 


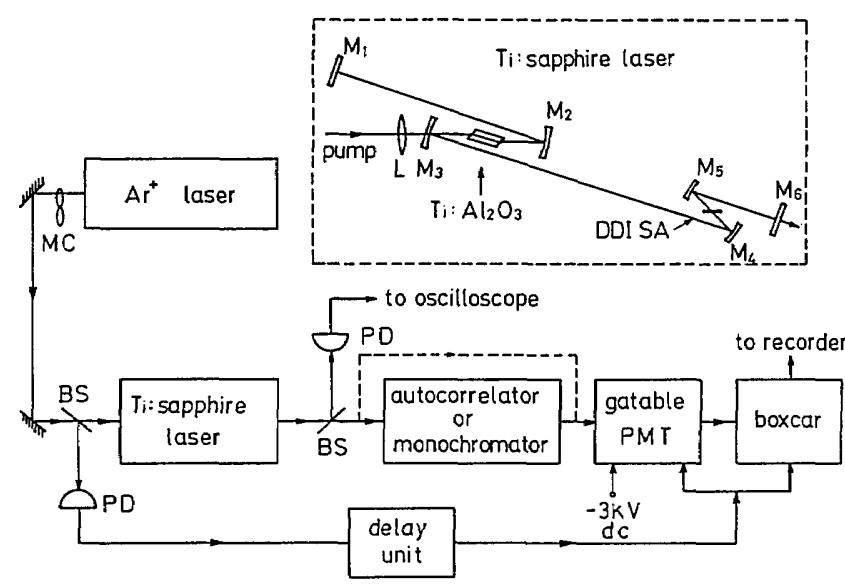

Fig. 1. Block diagram of the experimental arrangement. MC, mechanical chopper; BS's, beam splitters; PD's photodetectors; PMT, photomultiplier tube. The inset shows the configuration of the passively mode-locked Ti:sapphire laser: L, focal lens; SA, saturable absorber; $\mathrm{M}_{1}-\mathrm{M}_{6}$, mirrors.

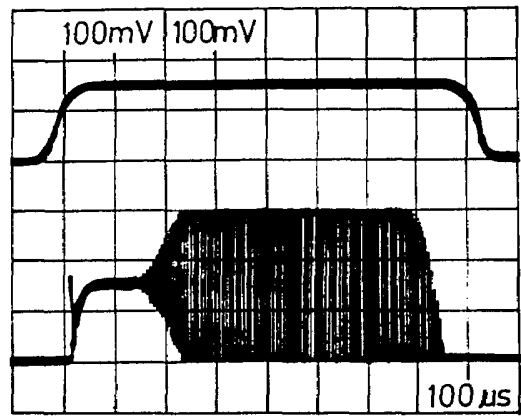

Fig. 2. Real-time display of the dynamics of pulse formation. The upper trace is the chopped argon-ion laser pump pulse with a FWHM of $800 \mu$ s and a rise time of $300 \mu \mathrm{s}$. The lower trace shows the pulse train buildup in the Ti:sapphire laser measured with a fast photodetector. The horizontal scale is $100 \mu \mathrm{s} /$ division.

tube (PMT). To sample the pulse evolution process, we controlled the PMT with a 50-ns gate to select five pulses. A boxcar integrator with a 100-ns gate has been used for signal averaging. By delaying these two synchronous gates with respect to the chopped pumping signal, we can sample the temporal and spectral characteristics of the Ti:sapphire laser as it evolves to the steady state. By feeding the output of the Ti:sapphire laser directly to the gatable PMT, we have also observed the evolution of its average output power.

A real-time oscilloscope display of the dynamics of pulse formation of the mode-locked Ti:sapphire/DDI laser as detected by a fast photodetector is shown in Fig. 2. Initially the saturable absorber just played the role of a loss element in the cavity. The laser output built up from spontaneous emission and operated in the cw stage in $\approx 140 \mu \mathrm{s}$. During the first $15 \mu \mathrm{s}$ of the $\mathrm{cw}$ regime, the laser exhibited relaxation oscillation at $200 \mathrm{kHz}$. As the circulating energy inside the cavity increased and saturated the DDI dye absorber, the output of the Ti:sapphire laser became modulated, and a pulse train appeared. In Fig. 3 we display the widths of the mode-locked pulses as a function of the delay time. This was measured by the time-gating method. Zero delay time was at the onset of Ti:sapphire laser action. The pulse width can be seen to shorten quickly during a period of $\approx 60 \mu \mathrm{s}$ after the laser output became modulated. In comparison, the shortening took $\approx 200 \mu \mathrm{s}$ in a mode-locked Ti:sapphire/HITCI laser with an intracavity compensating prism pair. ${ }^{14}$ This is explained as follows: At the starting stage of pulse development, the pulse width was longer and the chirping effect had not manifested itself. The additional prism pair would contribute an (as yet) uncompensated amount of group-velocity dispersion and be responsible for a longer buildup time than that of our Ti:sapphire/DDI laser without the compensating prism pair. Furthermore the system with the prism sequence eventually generated steady-state subpicosecond pulses and thus would require a longer buildup time than our picosecond laser without the compensating prism pair. The Ti:sapphire/DDI laser also exhibited an oscillatory behavior as it approached a steady-state value of $4.2 \mathrm{ps}$ in $\approx 370 \mu \mathrm{s}$. Excluding the initial $\mathrm{cw}$ operation regime, the buildup time to the steady state took $\sim 230 \mu \mathrm{s}$. The oscillatory behavior was not caused by the electronic artifacts (i.e., ringing), and similar behavior has previously been observed for a colliding-pulse mode-locked Rhodamine 6G/DODCI ring dye laser without the compensating prism sequence. ${ }^{17}$ Based on the similarities, we interpret the oscillation to be the result of combined action of the pulse-shortening forces and the pulse-broadening forces that are primarily due to group-velocity dispersion and to a lesser extent to self-phase modulation effects in the cavity. Also shown in Fig. 3 is the buildup of the average output power $P_{\mathrm{av}}$ of the Ti:sapphire laser. During the initial $30 \mu \mathrm{s}$, the rate of change of $P_{\mathrm{av}}$ was limited by the chopper. As $P_{\mathrm{av}}$ increased, this rate of change became faster once the laser pulse train was formed. Lower effective loss in the cavity owing to bleaching of the absorber was throught to be responsible for the faster rate. The steady-state value of $P_{\mathrm{av}}$ was reached in approximately the same time as that taken by the evolution of the pulse width.

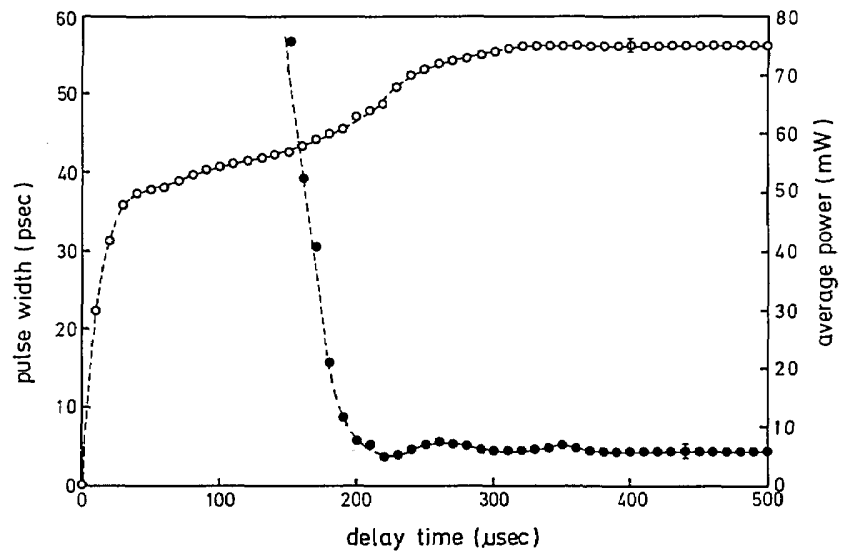

Fig. 3. Pulse width (filled circles) and average power (open circles) as a function of the delay time. Zero delay time corresponds to the onset of Ti:sapphire laser action. 


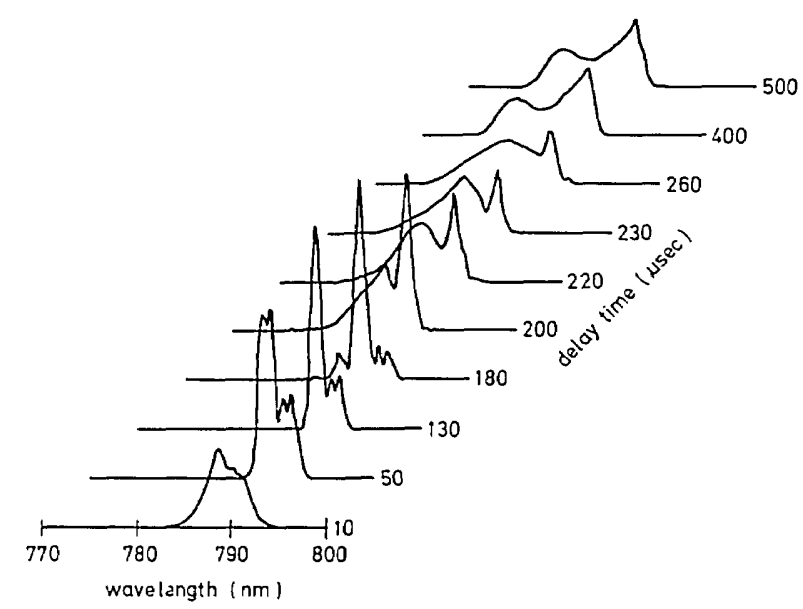

Fig. 4. Spectra of the output of the Ti:sapphire/DDI laser as a function of the delay time.

By increasing the pumping power of the argon-ion laser from 4.6 to $5.4 \mathrm{~W}$ with steps of $0.2 \mathrm{~W}$, we found that the duration in which the laser operated $\mathrm{cw}$ was shortened to $\approx 200,140,100,80$, and $60 \mu \mathrm{s}$, respectively. The pulse buildup times, starting from the time that the laser became modulated to the first minimum of oscillation of the pulse width, also shortened to $\approx 100,60,40,35$, and $30 \mu \mathrm{s}$, respectively. The shortening of these two characteristic times is attributed to the increase in the intracavity energy at higher pumping powers. The steady-state pulse widths, however, were nearly the same when the pumping power was varied in this range. It is not yet clear from our data and previous studies how the steady-state properties of passively mode-locked Ti:sapphire lasers are affected by the characteristics of a saturable absorber.

The evolution of the spectra of the laser output is shown in Fig. 4. Spectral narrowing was observed in the cw regime. This is reasonable, since the longitudinal modes would then be unlocked and random in phase, yielding a broad output spectrum. As the photon energy built up inside the cavity, the side modes ceased lasing and the bandwidth of the spectrum narrowed owing to gain competition among the longitudinal modes in a homogeneously broadened medium. This process took $\sim 110 \mu \mathrm{s}$. Once the laser began to operate in the mode-locked regime, the blue side of the spectrum gradually increased and the output spectrum broadened. A much longer pulse width was observed than was expected from the spectral width in the steady state (Fig. 4). This implies that the pulses show large chirping caused by the dispersion and self-phase modulation inside the Ti:sapphire rod. The gain saturation process will enhance the trailing-edge spectral component. As a result the observed spectrum exhibited a blue shift. A dramatically changed spectrum was also observed at the first minimum of oscillation of the pulse width. This behavior also resembled that of the colliding-pulse mode-locked Rhodamine 6G/DODCI ring dye laser without the compensating prism sequence. ${ }^{17}$

In summary, we have presented detailed experi- mental data on the dynamics of pulse formation in a cw passively mode-locked Ti:sapphire/DDI laser. This laser initially operates in a cw regime, becomes mode locked, and generates steady-state picosecond pulses in $\approx 370 \mu \mathrm{s}$. Excluding the cw stage, the pulse width rapidly shortens in $60 \mu \mathrm{s}$, and the entire pulse buildup process takes $\sim 230 \mu \mathrm{s}$. This corresponds to 23,000 round trips. An interesting oscillatory behavior during the evolution of the pulse width, attributed to the competition between the shortening forces and the broadening forces in the cavity, was also observed. Concurrently the bandwidth of the laser output spectrum initially narrowed owing to the competition among the longitudinal modes of the homogeneously broadened gain medium and broadened as the mode-locked pulse train evolved.

This research was partially supported by the National Science Council of the Republic of China under grants NSC79-0417-E009-04, NSC80-0417E009-05, and NSC80-0417-E009-17. The authors thank the reviewers for valuable comments, W.-D. Hwang and M.-H. Lu for the loan of equipment, and J. Wang for a preprint of his paper. Helpful discussions with Y. Lai are also acknowledged.

\section{References}

1. J. Goodberlet, J. Wang, J. G. Fujimoto, and P. A. Schulz, Opt. Lett. 14, 1125 (1989).

2. P. F. Curley and A. I. Ferguson, Opt. Lett. 16, 1016 (1991).

3. J. D. Kafka, M. L. Watts, D. J. Roach, M. S. Keirstead, H. W. Schaaf, and T. Baer, in Digest of Conference on Lasers and Electro-Optics (Optical Society of America, Washington, D.C., 1990), paper CPDP8.

4. U. Keller, W. H. Knox, and H. Roskos, Opt. Lett. 15, 1377 (1990).

5. D. E. Spence, P. N. Kean, and W. Sibbett, Opt. Lett. 16, 42 (1991).

6. J. P. Likforman, G. Grillon, M. Joffre, C. L. Blanc, A. Migus, and A. Antonetti, Appl. Phys. Lett. 58, 2061 (1991).

7. L. Spinelli, B. Couillaud, N. Goldblatt, and D. K. Negus, in Digest of Conference on Lasers and Electro-Optics (Optical Society of America, Washington, D.C., 1991), paper CPDP7.

8. N. Sarukura, Y. Ishida, H. Nakano, and Y. Yamamoto, Appl. Phys. Lett. 56, 814 (1990).

9. N. Sarukura, Y. Ishida, T. Yanagawa, and H. Nakano, Appl. Phys. Lett. 57, 229 (1990)

10. N. Sarukura, Y. Ishida, and H. Nakano, Opt. Commun. 77, 49 (1990).

11. N. Sarukura, Y. Ishida, and H. Nakano, Opt. Lett. 16, 153 (1991).

12. K. Naganuma and K. Mogi, Opt. Lett. 16, 738 (1991).

13. J. Goodberlet, J. Wang, J. G. Fujimoto, and P. A. Schulz, Opt. Lett. 15, 1300 (1990).

14. Y. Ishida, N. Sarukura, and H. Nakano, in Digest of Conference on Lasers and Electro-Optics (Optical Society of America, Washington, D.C., 1991), paper JMB2.

15. S. Chen and J. Wang, Opt. Lett. 16, 1689 (1991).

16. Exciton, Inc., laser dye catalog (Dayton, Ohio, 19891991), p. 33.

17. J. C. Kuo and C. L. Pan, Opt. Lett. 15, 1297 (1990). 